Conclusion In children and adolescents with T1DM DPN is highly prevalent, but in the majority of patients it is subclinical. Sensitivity and negative predictive values of the clinical neurological exam are low. Therefore, routine NCV measurement for the assessment of DPN appears warranted in these patients.

\section{EAST OR SESAME SYNDROME: A CASE SERIES}

doi:10.1136/archdischild-2012-302724.1513

DK Gandhi, R Gupta, S Philip, S Agrawal, E Wassmer. Paediatric Neurology, Birmingham Children's Hospital, Birmingham, UK

Introduction Molecular and genetic advances have changed the way we look at associations of signs and symptoms or miscellaneous syndromes. A recent report has hypothesized that KCNJ10 mutations, affecting potassium channels present in the brain, ear and the kidneys, are responsible for the constellation of epilepsy, ataxia, sensorineural deafness. and tubulopathy (EAST or SeSAME syndrome). We present six patients belonging to three families with similar findings.

Case Series We describe three Asian siblings, two Caribbean siblings, and one Caucasian child who have epilepsy, ataxia, sensorineural hearing loss, and tubulopathy. Consanguinity was present only in the Asian family. Seizures were a presenting symptom in four of the cases with onset as early as 3-7 months of age. Development delay and learning difficulties were present in all of the cases. Ataxia was evident from early on. Sensorineural hearing loss was identified at different ages and in some cases was asymptomatic. In some cases, tubulopathy was an incidental finding. Two of the children were being followed up by nephrologists and neurologists before a unifying diagnosis was determined. Five children had previously been extensively investigated with metabolic and mitochondrial investigations, magnetic resonance images, and electroencephalograms all normal. All six children had biochemical evidence of a tubulopathy with hypokalaemia, hypomagnesaemia, and alkalosis. KCNJ10 DNA mutations have been identified in all the children.

Conclusion Recent advances in genetics have enabled us to determine the likely unifying cause for hitherto puzzling signs and symptoms in six children under our care.

\section{DEMOGRAPHIC, ETIOLOGICAL AND CLINICAL CHARACTERISTICS OF OUR CEREBRAL PALSY CASES}

doi:10.1136/archdischild-2012-302724.1514

'E Aksoy, ${ }^{2} \mathrm{~S}$ Karasalihoğlu, ${ }^{2} \mathrm{Y}$ Karal, ${ }^{3} \mathrm{G}$ Ekuklu. ${ }^{1}$ Department of Pediatrics; ${ }^{2}$ Department of Pediatrics, Division Pediatric Neurology; ${ }^{3}$ Department of Public Health, Medical Faculty of Trakya University, Edirne, Turkey

Aim The aim of this study is to reveal demographic, etiological and clinical characteristics of our cerebral palsy cases and to underline differences peculiar to Thrace region of Turkey.

Methods One hundred and thirty five cerebral palsy cases, followed by Medical Faculty of Trakya University, Department of Pediatrics, Division Pediatric Neurology, were evaluated retrospectively. Data were obtained through out patient clinic files and patient discharge forms. Interviews by the phone were carried on in order to complete the necessary information. Demographic, etiological, laboratory and clinical characteristics of the cases were recorded.

Results The mean age of the cases was $112.65 \pm 47.02$ months (2-18 years) and boy/girl ratio was 1.8. The majority of the case etiologies were perinatal risk factors accounted for $61.5 \%$. Forty per cent of the cases were term AGA. Spastic type cerebral palsy constituted $91.9 \%$ of all cases with cerebral palsy while $46 \%$ of them were quadriplegic. Quadriplegic type was encountered most (46.2\%) in term deliveries, while diplegic type was the most common form in preterms (47.4\%). Speech problems $(77.8 \%)$ and mental retardation $(75.6 \%)$ were the most accompanying problems. Epilepsy accompanied $72.6 \%$ of the cases. An increasing rate of malnutrition was detected parallel to increasing age groups.

Conclusions Cerebral palsy cases showed certain differences in terms of demographic, etiological and clinical characteristics in Thrace region comparing to other regions.

\section{INFANTILE SPASM IN CHILDREN: CLINICAL FEATURES AND OUTCOME}

doi:10.1136/archdischild-2012-302724.1515

T Sultan, A Waheed Rathore. Child Neurology, Institute of Child Health, Lahore, Pakistan

Objective Clinical features and outcome of children with infantile spasms.

Study Design Interventional and observational study.

Place and Duration of Study The Department of Neurology, Children's Hospital, Lahore, Pakistan, from January 2010 to December 2011.

Methodology Children aged $<2$ years presented with history of infantile spasms were assessed. Clinical presentation, EEG findings and response of anti-epileptic drugs was analyzed.

Results A total of 51,370 children visited Neurology outpatient department of Children Hospital, Lahore. Out of them, 450 infants had infantile spasms at their first presentation. Mean age at presentation was $6.6+2.5$ months. Out of 450 children, $76 \%$ children presented at age $<6$ month, $72 \%$ presented due to infantile spasms and $18 \%$ because of global developmental delay. Spasm types were mixed (38\%), flexors (44\%), extensor $(16 \%)$ and asymmetric $(2 \%)$. Symptomatic seizures were seen in $72 \%$ and cryptogenic in $28 \%$. Hypsarrhythmia (67\%) was the predominant EEG finding followed by modified hypsarrhythmia (24\%) and other forms of epileptic discharges in $9 \%$ children. Majority of children were receiving oral Phenobarbitone, Carbamzaepine or Valproate sodium. We initiate the management with oral Prednisolone followed by Clonazepam or valproate acid. ACTH therapy was administered in only 5 children. Conclusion Infantile spasms are one of the refractory epilepsy in children. Abnormal EEG findings predominantly the hypsarrhythmia or modified hypsarrhythmia are the hallmark. Majority of children received conventional AED with poor response. Oral prednisolone is proved to be the most effective AED. These children should be referred to the tertiary care paediatric neurology centers.

\section{ELECTROPHYSIOLOGICAL STUDY OF PERIPHERAL NERVES IN CHILDREN WITH ACUTE LYMPHOBLASTIC LEUKEMIA}

doi:10.1136/archdischild-2012-302724.1516

M Imam. Physical Medicine, Faculty of Medicine - Alexandria University, Alexandria, Egypt

Introduction Acute lymphoblastic leukemia (ALL) is a malignant disorder of lymphoid progenitor cells, affects both children and adults. Chemotherapy induced peripheral neurotoxicity is an important side-effect of several chemotherapeutic agents.

The Aim of study was to detect the presence and types of peripheral neuropathy in newly diagnosed children with acute lymphoblastic leukemia, before and after the phase of induction of remission.

Patients and Methods 24 patients newly diagnosed as ALL were enrolled into the present study (group I); they were subjected to clinical and electrophysiological assessments which assess the integrity of the peripheral nervous system through conducting nerve conduction study and electromyography before and after receiving the chemotherapeutic agents. Ten apparently healthy children served as controls (group II).

Results 16 cases (67\%) out of the 24 examined ALL patients were proved electrophysiologically to be free from PN, while 8 cases 